\title{
Safety of Factor XIII Concentrate: Analysis of More than 20 Years of Pharmacovigilance Data
}

\author{
Cristina Solomon ${ }^{a}$ Wolfgang Korte $^{b}$ Dietmar Fries $^{c}$ Inna Pendrak ${ }^{d}$ Christine Joch $^{e}$ \\ Albrecht Gröner ${ }^{e}$ Ingvild Birschmann ${ }^{f}$ \\ ${ }^{a}$ Medical Affairs Acquired Bleeding Disorders, CSL Behring, Marburg, Germany; \\ ${ }^{b}$ Hemostasis and Hemophilia Center; and Center for Laboratory Medicine, St. Gallen, Switzerland; \\ ${ }^{\mathrm{c}}$ Department of Anesthesia and Intensive Care, Medical University Innsbruck, Innsbruck, Austria; \\ ${ }^{d}$ CSL Behring, King of Prussia, PA, USA;

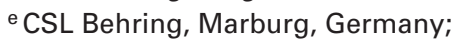 \\ ${ }^{f}$ Institute for Laboratory and Transfusion Medicine, Heart and Diabetes Center, Ruhr-University Bochum, Bad Oeynhausen, Germany
}

\section{Keywords}

Factor XIII · Pharmacovigilance · Safety

\section{Summary}

Background: Plasma-derived factor XIII (FXIII) concentrate is an effective treatment for FXIII deficiency. We describe adverse drug reactions (ADRs) reported during pharmacovigilance monitoring of Fibrogammin ${ }^{\circledR} /$ Corifact ${ }^{\circledR}$ and review published safety data. Methods: Postmarketing safety reports recorded by CSL Behring from June 1993 to September 2013 were analyzed. Clinical studies published during the same period were also reviewed. Results: Commercial data indicated that 1,653,450,333 IU FXIII concentrate were distributed over the review period, equivalent to 1,181,036 doses for a $70 \mathrm{~kg}$ patient. 75 cases were reported (one/15,700 standard doses or 22,046,000 IU). Reports of special interest included 12 cases of possible hypersensitivity reactions (one/98,400 doses or $137,787,500$ IU), 7 with possible thromboembolic events (one/168,700 doses or $236,207,200$ IU), 5 of possible inhibitor development (one/236,200 doses or 330,690,100 IU), and 20 of possible pathogen transmission (one/59,100 doses or $82,672,500$ IU). 19 pathogen transmission cases involved viral infection; 4 could not be analyzed due to insufficient data, but for all others a causal relationship to the product was assessed as unlikely. A review of published literature revealed a similar safety profile. Conclusion: Assessment of ADRs demonstrated that FXIII concentrate carries a low risk of ADRs across various clinical situations, suggesting a favorable safety profile.

(c) 2016 S. Karger GmbH, Freiburg

\section{Introduction}

Coagulation factor XIII (FXIII) is a pro-transglutaminase composed of two A subunits (FXIII- $\mathrm{A}_{2}$ ) and two B subunits (FXIII- $\mathrm{B}_{2}$ ), which plays an important role in hemostasis by stabilizing the blood clot and increasing its resistance to fibrinolysis. These effects are achieved by catalyzing the cross-linking of fibrin, platelet membrane, and matrix proteins $[1,2]$. Additionally, FXIII plays an important role in the promotion of angiogenesis and wound healing, and the maintenance of pregnancy $[1,2]$.

Congenital FXIII deficiency is a rare hereditary bleeding disorder estimated to affect one in every 1-5 million live births, which occurs with higher frequency in consanguineous families $[1,3-6]$. Patients suffering from FXIII deficiency can manifest severe bleeding conditions as early as a few days after birth, with umbilical cord bleeding occurring in $80 \%$ of cases of severe FXIII deficiency [3]. Additionally, patients carry a significant risk of intracranial hemorrhage, which occurs in $34 \%$ of patients and is the leading cause of death in this group $[3,5,7]$. Most patients with congenital FXIII deficiency suffer from life-long bleeding diathesis and impaired wound healing, and, as FXIII is one of the few coagulation factors that does not rise during pregnancy, complications and spontaneous abortion in pregnant women with FXIII deficiency are common $[2,3,5]$. Acquired FXIII deficiency is thought to be much more common than congenital deficiency [1]. Acquired FXIII deficiency could result as a consequence of hemorrhage or dilutional changes [6], from the development of inhibitory autoantibodies against FXIII $[1,5,6]$, or from diseases associated with overconsumption or reduced synthesis of FXIII $[5,6]$.

FXIII deficiency can be treated with cryoprecipitate or fresh frozen plasma (FFP); however, these treatments are generally avoided when other options are available as they carry a risk of

\section{KARGER}

() 2016 S. Karger GmbH, Freiburg

Fax +497614520714
Prof. Dr. Cristina Solomon 
transmitting blood-borne infections or increased risk of transfusion-related lung injury $[3,8,9]$. An alternative is treatment with plasma-derived FXIII concentrate. Fibrogammin ${ }^{\circledR}$ (CSL Behring $\mathrm{GmbH}$, Marburg, Germany) is a purified, heat-treated (pasteurized), FXIII concentrate derived from human plasma, which has been available in Europe since 1993. It is approved in Argentina, Austria, Belgium, Brazil, France, Germany, Great Britain, Indonesia, Israel, Japan, Luxembourg, and Switzerland, has also been approved by the US Food and Drug administration in 2011 and by Health Canada in 2013 under the trade name Corifact ${ }^{\circledR}$, and approval is ongoing in additional EU countries under the trade name Cluvot $^{\circledR}$.

Several studies have demonstrated that treatment with Fibrogammin/Corifact is an effective prophylactic and on-demand treatment for congenital FXIII deficiency, reducing the incidence of both spontaneous and postoperative bleeding $[4,10,11]$. Moreover, the FDA approval of Fibrogammin/Corifact for routine prophylaxis and perioperative bleeding management in congenital FXIII deficiency was based on the confirmed efficacy and safety profile established in 12 clinical trials with a total of 187 patients receiving 3,930 doses [12]. Fibrogammin/Corifact has also been shown to be efficacious in cases of acquired FXIII deficiency, such as reducing bleeding in ulcerative colitis [13], minimizing the incidence of myocardial edema during cardiac surgery in newborns and young children with congenital heart disease [14], reducing bleeding and transfusion requirements, and improving clot firmness during surgery [15-17], and reducing bleeding and red blood cell (RBC) transfusion requirements following stem cell transplantation $[18,19]$.

This descriptive analysis summarizes a large pharmacovigilance dataset for FXIII concentrate (Fibrogammin/Corifact) from 1993 to 2013 and reviews key postmarketing safety reports from the literature over the same time period. The intent is to investigate the safety profile of FXIII concentrate, including an evaluation of identified and potential risks, through the assessment of real-world data.

\section{Materials and Methods}

\section{Pharmacovigilance}

Adverse drug reaction (ADR) reports were compiled from June 1, 1993 to September 30, 2013 as part of the on-going CSL Behring routine pharmacovigilance. These included spontaneous reports, reports from regulatory agencies and cases identified from review of the worldwide scientific literature (all spontaneous reports were assumed to be ADRs). For each ADR report, the year, country of origin, patient age and sex, indication, FXIII concentrate dose, concomitant therapies, and manifestations and outcome of the ADR were recorded if available. Wherever possible, a narrative description of the ADR was obtained. Where relevant information was missing from the initial report, the reporting source was queried for further clarification; however, further information was not always available. ADRs were coded using the Medical Dictionary for Regulatory Activities (MedDRA) version 16.0, and the event was classified as serious versus non-serious according to the regulatory definition (i.e., a serious event was an event which resulted in death, was life-threatening, required hospitalization, resulted in persistent or significant disability/incapacity, was a congenital anomaly/birth defect, or was a medically important event) [20].

The cumulative quantity of FXIII concentrate distributed during the review period was established from CSL Behring commercial records. As the number of patients to whom the drug was distributed is not known, patient exposure is presented as number of estimated $20 \mathrm{IU} / \mathrm{kg}$ doses for a patient of $70 \mathrm{~kg}$ and per IU FXIII concentrate distributed. The reporting rate of cases involving ADRs is given to the nearest 100 doses or 100 IU.

Standardized MedDRA Queries (SMQs), High Level Group Terms (HLGT), High Level Terms (HLT), and Preferred Terms (PT) within the MedDRA dictionary were used as needed to identify cases of special interest, i.e., those including events considered to be important identified risks (anaphylaxis and hypersensitivity/allergic reactions and thromboembolic complications) or potential risks (suspicion of virus transmission, inhibitor development, and lack of effect) for analysis as follows:

- Anaphylaxis and hypersensitivity/allergic reactions were identified using the following SMQs: anaphylactic reactions (narrow); hypersensitivity (narrow).

- Thromboembolic complications were identified using the SMQ embolic and thrombotic events (broad).

- Suspicion of virus transmission was identified using the HLGT viral infectious disorders; the HLTs infectious transmissions, and virus identification and serology; and the PTs viremia, viral sepsis, and hepatitis post transfusion.

- Inhibitor development cases were identified using the following PTs as an SMQ does not exist: factor XIII inhibition; coagulation factor XIII level abnormal; coagulation factor XIII level decreased; coagulation factor inhibitor assay; acquired hemophilia with anti FVIII, XI, or XIII; inhibiting antibodies; antibody test positive.

- Lack of effect cases were identified using the following PTs as an SMQ does not exist: drug clearance increased; drug level below therapeutic; multiple drug resistance; device failure; device ineffective; drug effect decreased; drug effect delayed; drug effect incomplete; drug half-life reduced; drug ineffective; drug ineffective for unapproved indication; drug level decreased; drug resistance; drug-specific antibody present; drug tolerance; drug tolerance increased; multiple drug resistance; no therapeutic response; paradoxical drug reaction; tachyphylaxis; therapeutic product ineffective; therapeutic product ineffective for unapproved indication; therapeutic reaction time decreased; therapeutic response decreased; therapeutic response delayed; treatment failure; vaccination failure; virologic failure.

The cases retrieved using these SMQs and MedDRA terms were not reviewed to confirm that they met case definitions. Thus, it is likely that the total number of cases in each event of special interest category is an overestimate of the actual number of cases in that category.

The reporting rate of MedDRA PTs was assessed in all remaining reports that were not identified as involving events of special interest by the criteria listed above.

For pathogen transmission cases, causality assessment was also carried out. ADR reports were assessed for information regarding the causality of pathogen transmission, and the safety margin of Fibrogammin regarding the viruses in question was calculated according to the respective European Medicines Agency guideline (report no. EMA/CHMP/BWP/360642/2010 rev. 1) resulting in the statement '.. . The measures taken are considered effective for enveloped viruses such as human immunodeficiency virus (HIV), hepatitis B virus and hepatitis $\mathrm{C}$ virus and for the non-enveloped hepatitis A virus...' Nucleic acid amplification technique (NAT) was implemented for plasma donations at CSL Behring's predecessor companies after 1998, leading to all plasma pools for fractionation only being released for further manufacturing when non-reactive for genomic material of HCV (as per European guideline 'The introduction of nucleic acid amplification technology (NAT) for the detection of HCV RNA in plasma pools (CPMP/BWP/390/97)') and for genomic material of HIV and HBV accordingly. From March 2000 onward, only plasma donations and plasma pools for fractionation which were non-reactive for the non-enveloped HAV (and high titers of parvovirus B19) were approved for processing to the finished product Fibrogammin. Before the implementation of NAT, the manufacturing process of Fibrogammin ensured high virus reduction capacity through pasteurization and partitioning steps. However, in order to assess early cases of suspected virus transmission with Fibrogammin manufactured before the introduction of the NAT assays, vials of the final product were studied with a sensitive NAT assay (at least the same sensitivity as the assays used for the release of plasma pools for fractionation) and found non-reactive. 
Literature Review

A non-systematic literature search was conducted using MEDLINE (PubMed) with the objective of identifying original, English language articles published between June 1, 1993 and September 30, 2013, which reported on clinical studies of Fibrogammin/Corifact in bleeding patients. The following search string was used: ((('factor XIII' OR 'FXIII') AND ('concentrate' OR 'therapy' OR 'substitution')) OR 'corifact' OR 'fibrogammin' OR 'cluvot' OR 'Factor XIII/administration and dosage'[MeSH Terms] OR 'Factor XIII Deficiency/ therapy'[MeSH Terms] OR 'Factor XIII/adverse effects'[MeSH Terms] OR 'Factor XIII Deficiency/diagnosis'[MeSH Terms] OR 'Factor XIII Deficiency/ blood'[MeSH Terms] OR 'Factor XIII Deficiency/congenital'[MeSH Terms] OR 'Factor XIII Deficiency/complications'[MAJR] OR 'Factor XIII Deficiency/ drug therapy'[MAJR] OR 'Factor XIII/analysis'[MAJR] OR 'Factor XIII/therapeutic use'[MAJR]).

The clinical study subjects, authors, methods, and time periods were examined to avoid inclusion of redundant data from multiple reports or data that had already been reported as part of the pharmacovigilance. The following data were extracted from the study reports: number and age of subjects, indication for FXIII concentrate infusion, treatment regimen, and occurrence of adverse events (AEs). Only studies reporting safety data were included, and all AEs reported in the clinical studies were included regardless of whether or not a relationship to FXIII concentrate was established. All study types were included and, in order to focus the review on clinical trials/studies, case reports/series with fewer than 15 cases were excluded; the published literature is regularly checked and case reports are added to the pharmacovigilance database as described above. As such, published case reports will have already been included as part of the pharmacovigilance analysis.

\section{Results}

\section{Pharmacovigilance}

\section{Baseline Characteristics}

Between June 1, 1993 and September 30, 2013, a total of 219 events in 75 cases were reported. The gender ratio was approximately even (37 male vs. 35 female, gender was not recorded in three cases). The patients' median (range) age was 50.5 (0-81) years. A total of six fatal cases were reported. 26 cases (34.7\%) were reported in congenital FXIII deficiency patients, and 17 cases $(22.7 \%)$ were reported as receiving ongoing prophylactic treatment with FXIII concentrate at the time of the ADR.

\section{Nature and Rate of Adverse Drug Reactions}

During the reporting period 1,653,450,333 IU of FXIII concentrate were distributed, equivalent to 1,181,036 doses assuming an average dose of $20 \mathrm{IU} / \mathrm{kg}$ for a patient of $70 \mathrm{~kg}$. As 75 cases were reported, this corresponds to approximately one case reported for every 15,700 doses administered or $22,046,000$ IU distributed. Of these, 54 cases were classified as serious according to regulatory definition, corresponding to approximately 1 serious case reported for every 21,900 doses or 30,619,500 IU distributed.

\section{Anaphylaxis and Hypersensitivity/Allergic Reactions}

A total of 12 cases (16.0\% of all cases) with 15 possible anaphylactic or hypersensitivity reaction events were reported, corresponding to approximately 1 case reported for every 98,400 doses or $137,787,500 \mathrm{IU}$ distributed. The median (range) age of the pa- tients was $63.5(0.8-78)$ years. The gender ratio was even, with 6 male and 6 female patients. Three of the reported cases (25.0\%) were in congenital FXIII deficiency, and $1(8.3 \%)$ of the cases was receiving ongoing prophylactic treatment with FXIII concentrate at the time of the ADR. None of the cases reported had a fatal outcome. The most common MedDRA PTs reported were hypersensitivity, rash, and rash generalized ( 3 cases each). The use of concomitant drugs was recorded in 9 of the 12 cases (75\%).

\section{Thromboembolic Complications}

A total of 7 cases (9.3\% of all cases) with 14 possible thromboembolic events (TEEs) were reported (table 1); this is approximately 1 case reported for every 168,700 doses or 236,207,200 IU distributed. Three of the patients were male, and 4 were female. The median (range) age was 48.0 (0-69) years. Three of the reported cases $(42.9 \%)$ were in congenital FXIII deficiency, and 4 (57.1\%) of the cases were receiving ongoing prophylactic treatment with FXIII concentrate at the time of the ADR. Two of the cases have been previously published $[21,22]$, and 1 case had a fatal outcome (table 1, case no. 4). The most common MedDRA PTs reported were deep vein thrombosis and pulmonary embolism (2 cases each). In all cases, additional risk factors existed that provide alternative causes for the TEE (table 1), such as concomitant treatment with other coagulation factors (in 3 cases, including plasma, cryoprecipitate, and/or whole blood) or relevant medical history and concomitant diseases (in all cases, including migraine, hypertension, unstable angina, recent surgery, cancer, port catheter change, and existing deep vein thrombosis). The majority of cases were reported in acquired FXIII deficiency (71.4\%, 5 cases).

\section{Suspicion of Pathogen Transmission}

A total of 20 cases (26.7\% of all cases) with 26 possible events of transmission of pathogens were reported; this is approximately 1 case for every 59,100 doses or 82,672,500 IU distributed. One of these cases involved a possible hypersensitivity reaction and is also included above. The median (range) age was $52.0(0.8-77)$ years. There were more male patients than female ( $12 \mathrm{vs.} 7$; gender was not recorded in 1 case), and 1 case had a fatal outcome. Two of the reported cases $(10.0 \%)$ were in congenital FXIII deficiency, and none of the cases were receiving ongoing prophylactic treatment with FXIII concentrate at the time of the ADR. Of the 20 cases reported, 1 involved a case of bacterial pharyngitis with symptoms of cough, fever, and throat swelling; the remaining 19 cases all involved viral infections. For the bacterial case, a causal relationship to the product was assessed as unlikely, as validated sterile filtration of the final product excludes Fibrogammin as the source of the bacterial infection. The suspected virus transmission cases included 13 suspected cases related to HCV (including 1 case with suspected HAV transmission, with no confirmation of hepatitis A infection at the time of treatment), 5 suspected cases related to $\mathrm{HBV}$, and one suspected case related to HAV (in addition to the case mentioned above). In all cases concerning suspected virus transmission (except 4 with insufficient data) a causal relationship to the product was assessed as unlikely due to an alternative expla- 
Table 1. Reported cases involving thromboembolic complications

\begin{tabular}{|c|c|c|c|c|c|c|}
\hline Case no. & Indication & $\begin{array}{l}\text { Event } \\
\text { (preferred term) }\end{array}$ & $\begin{array}{l}\text { Concomitant and } \\
\text { co-suspect drugs }\end{array}$ & \multicolumn{2}{|l|}{ Gender } & Confounding factors \\
\hline \multicolumn{7}{|c|}{ Congenital FXIII deficiency } \\
\hline 1 & Migraine/headache & $\begin{array}{l}\text { Monoplegia } \\
\text { Monoparesis }\end{array}$ & - & $\mathrm{F}$ & & $\begin{array}{l}\text { Relevant history: experienced transient headaches } \\
\text { during previous year (no family history of migraine) } \\
\text { Due to lack of clinical or biological evidence for } \\
\text { hemorrhagic or thrombosis etiology a final diagnosis of } \\
\text { associated migraine was given }\end{array}$ \\
\hline $2[21]$ & $\begin{array}{l}\text { Congenital severe } \\
\text { FXIII deficiency }\end{array}$ & $\begin{array}{l}\text { Acute myocardial } \\
\text { infarction } \\
\text { Coronary artery } \\
\text { occlusion }\end{array}$ & $\begin{array}{l}\text { Whole blood, } \\
\text { cryoprecipitate, } \\
\text { clopidogrel, } \\
\text { simvastatin, } \\
\text { candesartan, } \\
\text { thiazide }\end{array}$ & M & & $\begin{array}{l}\text { Relevant history: episode of unstable angina, } \\
70 \% \text { stenosis of the left circumflex coronary artery } \\
\text { Concomitant disease: easy bruising, abnormal urea clot } \\
\text { lysis test, hypertension, moderate midwaist obesity, } \\
\text { mild hyperlipidemia } \\
\text { Risk factor: family history of coronary artery disease } \\
\text { (CAD) }\end{array}$ \\
\hline \multicolumn{7}{|c|}{ Acquired FXIII deficiency } \\
\hline 3 & $\begin{array}{l}\text { Postoperative su- } \\
\text { ture insufficiency }\end{array}$ & $\begin{array}{l}\text { Disseminated in- } \\
\text { travascular coag- } \\
\text { ulation }\end{array}$ & $\begin{array}{l}\text { Famotidine, } \\
\text { piperacillin sodium, } \\
\text { gabexate mesilate, } \\
\text { FFP }\end{array}$ & $\mathrm{F}$ & 52 & $\begin{array}{l}\text { Relevant history: pylorus-preserving } \\
\text { pancreaticoduodenotomy, gastropancreatic anastomosis, } \\
\text { cholangiojejunostomy, duodenojejunostomy } \\
\text { Concomitant disease: hypertension, common bile duct } \\
\text { carcinoma }\end{array}$ \\
\hline 4 & $\begin{array}{l}\text { Persistent chylo- } \\
\text { thorax, pleural ef- } \\
\text { fusion }\end{array}$ & $\begin{array}{l}\text { Embolism }^{*} \\
\text { Cerebral artery } \\
\text { embolism } \\
\text { Shunt occlusion* }\end{array}$ & $\begin{array}{l}\text { Packed RBC, FFP, } \\
\text { platelets, nutritional } \\
\text { support, lasix, } \\
\text { dobutamine, } \\
\text { buminate }\end{array}$ & M & 7 days & $\begin{array}{l}\text { Relevant history: cardiac operation, General anaesthesia } \\
\text { Concomitant diseases: asplenia syndrome, univentricular } \\
\text { heart/single atrium, total anomalous pulmonary venous } \\
\text { connection (at birth), pulmonary congestion (at birth) }\end{array}$ \\
\hline 5 & $\begin{array}{l}\text { Deficiency identi- } \\
\text { fied in congenital } \\
\text { afibrinogenemia } \\
\text { patient }\end{array}$ & $\begin{array}{l}\text { Venous } \\
\text { thrombosis limb }\end{array}$ & Haemocomplettan P & $\mathrm{F}$ & 20 & Concomitant disease: port catheter change \\
\hline 6 & IV injection & $\begin{array}{l}\text { Deep vein } \\
\text { thrombosis } \\
\text { Pulmonary } \\
\text { embolism }\end{array}$ & - & $\mathrm{F}$ & 48 & $\begin{array}{l}\text { Relevant history: deep vein thrombosis popliteal left, } \\
\text { factor V Leiden mutation } \\
\text { Concomitant disease: severe acquired FXIII deficiency, } \\
\text { post-thrombotic syndrome, HAV positive, hereditary de- } \\
\text { ficiency of other clotting factors }\end{array}$ \\
\hline $7[22]$ & $\begin{array}{l}\text { Severe hemorrhagic } \\
\text { diathesis }\end{array}$ & $\begin{array}{l}\text { Pulmonary } \\
\text { embolism } \\
\text { Deep vein } \\
\text { thrombosis } \\
\text { Device occlusion }\end{array}$ & - & M & 65 & $\begin{array}{l}\text { Relevant history: partial gastrectomy for an ulcer } \\
\text { Concomitant disease: massive hemorrhages, severe } \\
\text { acquired FXIII deficiency, IgG anti-factor XIII inhibitor, } \\
\text { hypertension, hyperthyroidism }\end{array}$ \\
\hline
\end{tabular}

nation. For the majority of cases, a causal relationship to the product was assessed as unlikely due to the safety margin of Fibrogammin regarding the viruses in questions, calculated as described in the methods. One or more of the following confounding factors were identified in all 17 cases with available data: no information available regarding the respective viral infection prior to treatment, preventing assessment as to whether the infection occurred prior to or after FXIII concentrate administration ( 9 cases); blood components (e.g. RBC, FFP, platelets) were administered concomitant with FXIII concentrate administration (15 cases); based on the parameters obtained during diagnosis of the respective viral infec- tion, the infection was contracted either prior to or 4 years after the treatment with FXIII concentrate $(8$ cases, these parameters include primarily specific serological markers with a known interval between infection and appearance or elevated liver enzymes related to this parameter). From further examination of the cases, 1 case of HBV transmission was considered to be most probably due to a concomitant plasma-derived product [23], and one case of HAV transmission was considered to be due to contaminated food (berries) mentioned as a probable source of infection in the report; furthermore, the HAV infection occurred considerably later than the treatment with FXIII concentrate. 
Fig. 1. Events observed in three or more reported postmarketing cases not classified as anaphylactic, allergic, thromboembolic, virus-related, or involving inhibitor development.

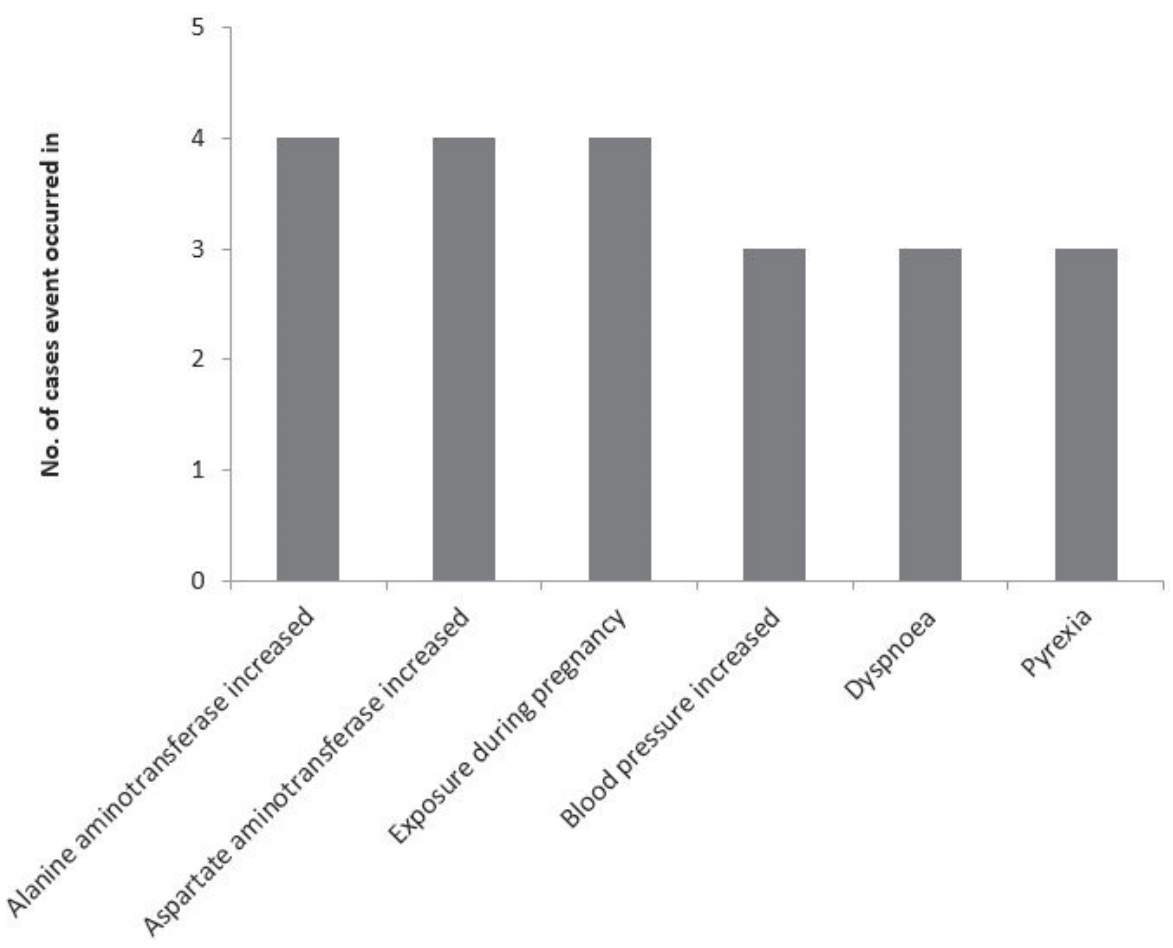

Adverse event (preferred term)

\section{Inhibitor Development}

A total of 5 cases (6.7\%) with 6 possible inhibitor development events were reported; this is approximately 1 case reported for every 236,200 doses or 330,690,100 IU distributed. Inhibitors were detected either by using a dot blot test (3 cases) or a laboratory test based on blood clot incubation (1 case); information was not available regarding inhibitor detection in the 5 th case. No specific information was provided on whether the inhibitors were permanent or transient. The median (range) age of the patients was 31.0 (1.8-74) years, and there were 2 male and 3 female patients. All 5 of the reported cases $(100.0 \%)$ were in congenital FXIII deficiency, and 3 (60.0\%) of the cases were receiving ongoing prophylactic treatment with FXIII concentrate at the time of the ADR. Two patients received immunomodulatory/immunosuppressive treatment, and 2 patients received immune tolerance therapy for the treatment of inhibitors. In 2 cases, plasma had been previously administered for prophylaxis or therapy of bleeding, and in 1 of these 2 cases an inhibitor antibody for FXIII had developed following treatment with plasma. This latter case had a fatal outcome, following fever with respiratory distress that progressed to septic shock and death. The physician reporting the case assessed the event as not related to FXIII concentrate.

\section{Lack of Effect}

A total of 6 cases ( $8.0 \%$ of all cases) with 6 events regarding lack of effect were reported; this is approximately 1 case for every 196,800 doses or $275,575,100$ IU distributed. The median (range) age of the patients was $34.0(20-65)$ years, and there were 4 male and 2 female patients. Three of the reported cases (50.0\%) were in congenital FXIII deficiency, and 2 (33.3\%) of the cases were receiv- ing ongoing prophylactic treatment with FXIII concentrate at the time of the ADR. Fibrogammin was reported as ineffective in 4 cases (including 1 case in conjunction with inhibitor development, and 1 case in conjunction with a TEE); in addition, 2 cases reported that the drug effect was incomplete.

\section{Other Reported Events}

28 cases were not classified as anaphylactic, allergic, thromboembolic, virus-related, or involving inhibitor development or lack of effect. When assessing these cases by the MedDRA System Organ Class (SOC), the most common type of events were investigations (11 cases), general disorders and administration site conditions (10 cases) and injury, poisoning and procedural complications ( 8 cases); a case may have more than 1 event. The most common MedDRA PTs reported (in 3 or more cases) were alanine aminotransferase increased, aspartate aminotransferase increased, exposure during pregnancy, blood pressure increased, dyspnea, and pyrexia (fig. 1).

\section{Literature Review}

Fibrogammin/Corifact has been administered in 8 postmarketing clinical studies that reported safety results between June 1, 1993 and September 30, 2013. One of the papers was excluded from the literature search results as it described cases that are included in the pharmacovigilance results (Matayoshi et al. [24]). The results of the remaining seven studies are summarized in table 2 .

FXIII concentrate was administered to 158 patients for a range of indications covering both congenital and acquired FXIII deficiency (in addition, one study included 22 patients, but did not state how many received FXIII concentrate [15]). The majority of 


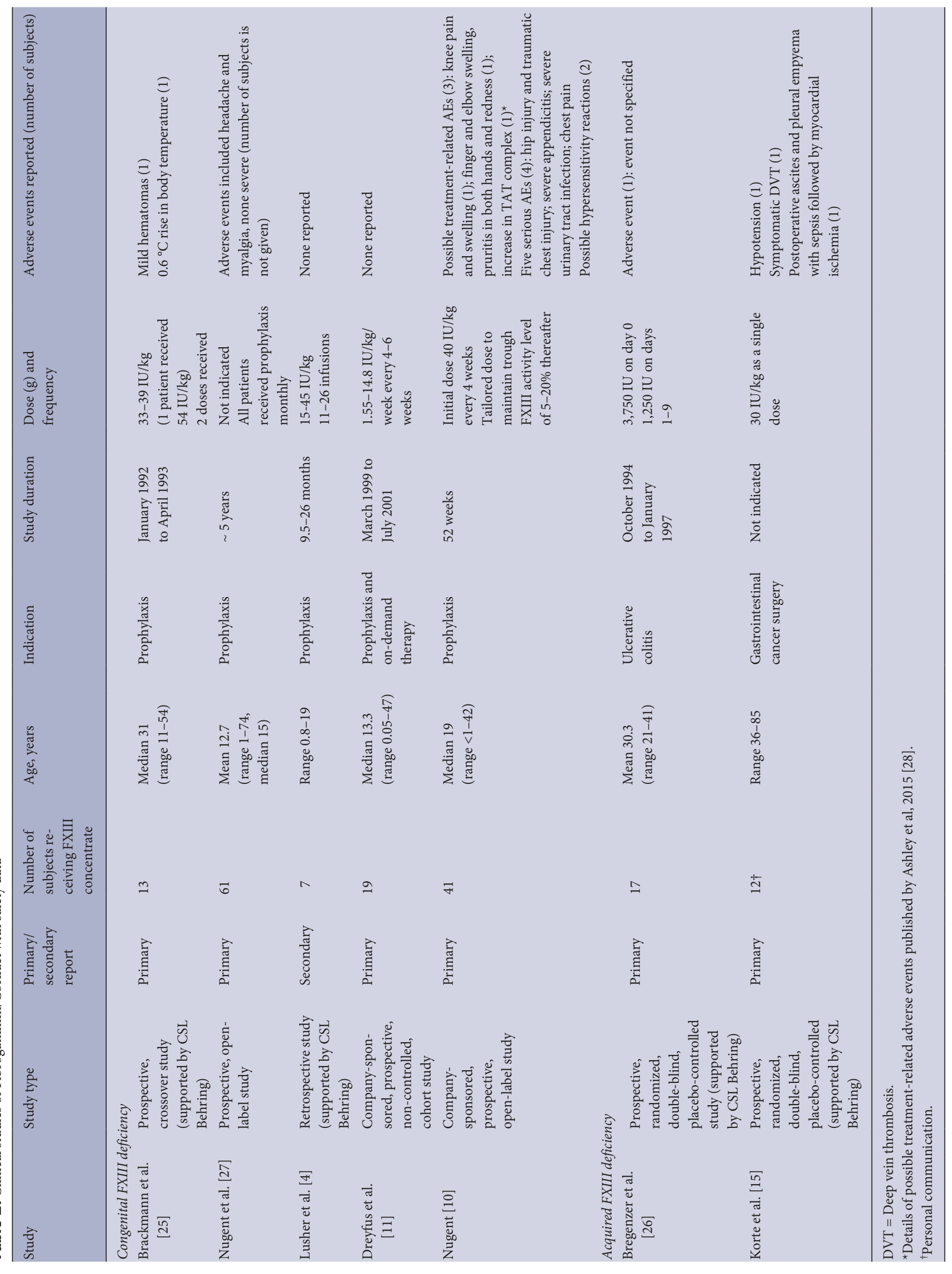


the studies were prospective $[10,11,15,25-27]$, and one was retrospective [4]. The number of patients in each study ranged from 7 to 61 . Both adult and pediatric patients were included in five studies $[4,10,11,25,27]$; the rest included patients $\geq 16$ years old $[15,26]$.

Two of the studies reported that no AEs were observed and are not discussed below $[4,11]$.

\section{Company-Sponsored Studies}

A prospective, open-label study of the efficacy and safety of Fibrogammin/Corifact for prophylactic treatment of congenital FXIII deficiency was published by Nugent [10]. In this study, 41 subjects received FXIII concentrate, and there were no reports of virus transmission or TEE. In the preliminary report, the total number of AEs was not reported; however, possible treatment-related AEs were reported in 3 subjects [10]. The full data was published in 2015, and clarified the possible treatment-related AEs as knee pain and swelling, finger and elbow swelling, pruritis in both hands and redness, and an increase in TAT complex [28]. In addition, 4 subjects experienced 5 serious AEs (hip injury, traumatic chest injury, severe appendicitis, severe urinary tract infection, and chest pain) which were judged unrelated or unlikely to be related to study medication [10]. Two subjects also reported possible hypersensitivity reactions: 1 mild reaction following treatment administration and 1 case of hives following prophylactic administration of FFP for an emergency appendectomy.

\section{Studies Supported by the Company}

A prospective crossover study to evaluate the efficacy and tolerability of plasma-derived versus placenta-derived FXIII concentrate in congenital FXIII deficiency was carried out by Brackmann et al. [25] in 13 subjects. No serious AEs were observed, and 2 minor AEs possibly related to the study medication were reported in subjects receiving plasma-derived FXIII (mild hematomas and $0.6{ }^{\circ} \mathrm{C}$ rise in body temperature), compared with 3 minor AEs in the subjects receiving placenta-derived FXIII (mild pruritus and erythema, slight pressure in the throat, and headache). No therapy was required to treat these AEs.

Bregenzer et al. [26] carried out a randomized, double-blind study to assess the efficacy of Fibrogammin in reducing the symptoms (particularly intestinal bleeding) of ulcerative colitis. No serious AEs were reported; AEs were reported in one of the 17 subjects in the FXIII group and in 2 of the 11 subjects in the placebo group (the nature of the AEs was not specified).

Korte et al. [15] reported a prospective, randomized, proof-ofconcept study comparing treatment with Fibrogammin or placebo to improve clot firmness in patients undergoing elective gastrointestinal cancer surgery. Following administration of FXIII concentrate, AEs were observed in 3 of the 12 subjects receiving FXIII concentrate. One episode of hypotension was observed in a subject with a history of hypotension. One subject developed a symptomatic deep vein thrombosis 7 days after extensive ( $>10 \mathrm{~h}$ ) surgery. Given the length and extent of surgery, the concomitant administration of blood products $(9,800 \mathrm{ml}$ of RBCs, platelets and FFP), and the fact that the subject's course of FXIII activity during sur- gery was below or in the low normal range, the authors concluded that the AE was unlikely to be related to FXIII concentrate administration. The final subject was a diabetic who developed postoperative ascites and pleural empyema with sepsis, followed by a myocardial ischemia 30 days later. Given that the subject's intraoperative course of factor XIII activity was below the normal range, the postoperative complications (including pleural empyema) that occurred between surgery and myocardial ischemia, and the time delay between drug administration and the ischemia, the authors concluded that this event was unlikely to be related to FXIII concentrate administration.

\section{Independent Studies}

A long-term (almost 5 years at time of publication) prospective study of prophylaxis with FXIII concentrate in congenital FXIII deficiency was carried out by Nugent et al. [27], and interim data in 61 subjects was published in 2006. AEs reported included headache and some myalgias (number of events was not given); however, none of the events were severe [27]. Two deaths were recorded, both unrelated to the underlying bleeding disorder or to FXIII replacement.

\section{Discussion}

This descriptive analysis of over 20 years of pharmacovigilance data indicates that the rate of ADRs following the administration of Fibrogammin/Corifact across diverse clinical settings is low. In total 75 cases were reported, corresponding to a rate of 1 case for every 15,700 standard doses of $20 \mathrm{IU} / \mathrm{kg}$ for a patient of $70 \mathrm{~kg}$ body weight or for every 22,046,000 IU FXIII concentrate distributed.

One of the concerns associated with administering a coagulation factor replacement product is the perceived risk of triggering a TEE. We only identified 7 cases reporting a possible TEE, corresponding to 1 case reported for every 168,700 standard doses or 236,207,200 IU distributed, and in all cases assessment was confounded by multiple risk factors for TEEs. This low rate of TEEs is supported by the literature review carried out here, and by a recent literature review of FXIII deficiency management, which did not observe any TEEs in the articles examined [3].

Although rare, the development of inhibitory antibodies against FXIII in patients receiving FXIII supplementation has been observed $[1,29,30]$. The development of inhibitors to FXIII in congenital patients would considerably complicate management of the patient; therefore, the incidence of inhibitor development was also evaluated. A low rate of inhibitor development was observed, with a total of 5 cases reported, corresponding to 1 case for every 236,200 doses or 330,690,100 IU distributed. This low rate is supported by the findings of a recent literature review, which concluded that the incidence of inhibitor development was rare [3]. A rare bleeding disorder registry created in North America observed inhibitor development in 3\% of patients receiving FXIII concentrate [31]. While low, this appears to be a higher rate than observed in our analysis; however, our analysis considers the rate of inhibi- 
tor development per dose, rather than per patient (as in the registry), making it difficult to directly compare these data.

The transmission of viruses is a known risk of plasma-derived products, although the manufacturing process for Fibrogammin/ Corifact includes virus inactivation and removal steps, involving pasteurization at $60^{\circ} \mathrm{C}$ for $10 \mathrm{~h}$, adsorption of $\mathrm{Al}(\mathrm{OH})_{3} / \mathrm{Vitacel}$ and defibrination, ion-exchange chromatography, and, since 2012, virus filtration employing two filters in series with a mean pore size of $19 \mathrm{~nm}$ each [10,32]. In addition to the virus removal and/or inactivation measures applied during the production process, prevention of transfusion of blood-borne viruses also includes steps related to limiting the virus input to the human plasma used as source [33], including donor selection and screening of individual donations and plasma pools for specific markers of infection. In this review of the pharmacovigilance database, 19 cases were identified involving suspected transmission of viral infectious agents. However, given that further analysis revealed non-reactive NAT tests for the concerned batches and related plasma pools for fractionation, and considering the virus removal/inactivation steps during the manufacturing process, these cases were assessed as not likely to be associated with FXIII concentrate administration.

Apart from FXIII concentrates, further alternatives for supplementing FXIII levels focus on the infusion of therapeutic plasma (e.g. FFP) or cryoprecipitate. However, these treatments carry their own risks, such as the potential to transmit blood-borne infections, allergic reactions, citrate toxicity, transfusion-related lung injury or transfusion-associated cardiac overload $[8,9,30,34]$. Indeed, cryoprecipitate has been withdrawn from a number of European countries due to safety concerns [35].

Analysis of the study results reported in the scientific literature indicated that the number of AEs observed was generally low, in agreement with the results of the pharmacovigilance database. Assessment of treatment-related complications in a rare bleeding disorder registry in North America and in a literature review of FXIII deficiency management also observed a low rate of AEs, supporting our results [3, 31]. Moreover, no virus transmission or inhibitor development events were identified during the literature review, and there were no unusual patterns of AEs reported that are not described in the product label.

Although postmarketing pharmacovigilance is a valuable tool for identifying potential risks from the available safety data, there are limitations, such as the potential underreporting of ADRs. As reporting of ADRs during pharmacovigilance is a voluntary process, the available data is limited by the willingness of the reporting source to provide the case and to supply any supporting information. Indeed, one review has suggested that the level of underreporting may be as high as $90 \%$ [36]. Healthcare professionals may be more likely to submit an ADR when it is assessed as serious, and this suggestion is supported by our finding that the majority (72\%) of the ADRs received were considered to be serious. Moreover, many of the patients who received FXIII concentrate also received other therapies which may have contributed to the development of any ADRs observed, further complicating the assessment of a case and establishing its relationship to FXIII concentrate. Additionally, there may be differences in reporting requirements between countries, and there may be cultural differences which make the reporting of spontaneous cases more or less likely in different countries. Another limitation of this analysis is that the precise exposure data is not known, and so approximations based on units distributed or estimated dosage have to be used. This makes it difficult to draw direct comparisons with published study data. In its favor, however, postmarketing reports allow for the assessment of 'real-world' data taken from large-scale and comprehensive coverage of the patient population. Additionally, as the cases retrieved using SMQs and MedDRA terms were not reviewed to confirm that they met case definitions, it is likely that the ADRs included in each event of special interest category represent an overestimate of the actual number of cases in that category.

Although this review goes some way towards providing useful safety data on the use of FXIII concentrate, there is a need for prospective studies of sufficient size and long-term follow-up to assess the incidence of rare ADRs. Nevertheless, this assessment of over 20 years of real-world data indicates that treatment with FXIII concentrate carries a low risk of ADRs.

\section{Acknowledgements}

The investigation was funded by CSL Behring. Editorial assistance with manuscript preparation was provided by Meridian HealthComms and funded by CSL Behring.

\section{Disclosure Statement}

CS, IP, CJ, and AG are employees of CSL Behring.

WK has received speaker fees, travel support and research support from CSL Behring, Novo Nordisk and Baxter.

DF has received study funding, payments, and travel funding from Austrian National Bank, AOP Orphan, Astra Zeneca, Baxter, B. Braun, Biotest, CSL Behring, Fresenius Kabi, Glaxo, Haemoscope, Hemogem, Lilly, LFB, Mitsubishi Pharma, Novo Nordisk, Octapharma, and TEM International.

IB has acted as paid speaker in the past for Siemens Healthcare Diagnostics Products $\mathrm{GmbH}$, Instrumentation Laboratory $\mathrm{GmbH}$ and CSL Behring $\mathrm{GmbH}$, received funding for research from Siemens Healthcare Diagnostics Products GmbH, Baxter Deutschland GmbH, Novo Nordisk Health Care AG and CSL Behring $\mathrm{GmbH}$ and travel expenses from Octapharma $\mathrm{GmbH}$, Novo Nordisk Health Care AG, Baxter Deutschland GmbH, Pfizer Pharma GmbH, SanofiAventis Deutschland GmbH and CSL Behring GmbH. 


\section{References}

1 Hsieh L, Nugent D: Factor XIII deficiency. Haemophilia 2008;14:1190-1200.

2 Muszbek L, Bereczky Z, Bagoly Z, Komaromi I, Katona E: Factor XIII: a coagulation factor with multiple plasmatic and cellular functions. Physiol Rev 2011;91 931-972.

3 Odame JE, Chan AK, Wu JK, Breakey VR: Factor XIII deficiency management: a review of the literature. Blood Coagul Fibrinolysis 2014;25:199-205.

4 Lusher J, Pipe SW, Alexander S, Nugent D: Prophylactic therapy with Fibrogammin $\mathrm{P}$ is associated with a decreased incidence of bleeding episodes: a retrospective study. Haemophilia 2010;16:316-321.

5 Fadoo Z, Merchant Q, Rehman KA: New developments in the management of congenital factor XIII deficiency. J Blood Med 2013;4:65-73.

6 Levy JH, Greenberg C: Biology of factor XIII and clinical manifestations of factor XIII deficiency. Transfusion 2013;53:1120-1131.

7 Ivaskevicius V, Seitz R, Kohler HP, Schroeder V, Muszbek L, Ariens RA, Seifried E, Oldenburg J, Study Group: International registry on factor XIII deficiency: a basis formed mostly on European data. Thromb Haemost 2007;97:914-921.

8 Murad MH, Stubbs JR, Gandhi MJ, Wang AT, Paul A, Erwin PJ, Montori VM, Roback JD: The effect of plasma transfusion on morbidity and mortality: a systematic review and meta-analysis. Transfusion 2010; 50:1370-1383.

9 O'Shaughnessy DF, Atterbury C, Bolton Maggs P, Murphy M, Thomas D, Yates S, Williamson LM, British Committee for Standards in Haematology BTTF: guidelines for the use of fresh-frozen plasma, cryoprecipitate and cryosupernatant. Br J Haematol 2004;126:11-28.

10 Nugent D: Corifact/Fibrogammin ${ }^{\circledR} \mathrm{P}$ in the prophylactic treatment of hereditary factor XIII deficiency: results of a prospective, multicenter, open-label study. Thromb Res 2012;130(suppl 2):S12-14.

11 Dreyfus M, Barrois D, Borg JY, Claeyssens S, Torchet MF, Arnuti B, Pautard B; Groupe d'Etudes Francophone du FXIII: Successful long-term replacement therapy with FXIII concentrate (Fibrogammin ${ }^{\circledR} \mathrm{P}$ ) for severe congenital factor XIII deficiency: a prospective multicentre study. J Thromb Haemost 2011;9:1264-1266.

12 James AH, Kouides PA, Abdul-Kadir R, Dietrich JE, Edlund M, Federici AB, Halimeh S, Kamphuisen PW, Lee CA, Martinez-Perez O, McLintock C, Peyvandi F, Philipp C, Wilkinson J, Winikoff R: Evaluation and management of acute menorrhagia in women with and without underlying bleeding disorders: consensus from an international expert panel. Eur J Obstet Gynecol Reprod Biol 2011;158:124-134.
3 Lorenz R, Born P, Olbert P, Classen M: Factor XIII substitution in ulcerative colitis. Lancet 1995;345:449450.

14 Wozniak G, Noll T, Akinturk H, Thul J, Muller M: Factor XIII prevents development of myocardial edema in children undergoing surgery for congenital heart disease. Ann N Y Acad Sci 2001;936:617-620.

15 Korte WC, Szadkowski C, Gahler A, Gabi K, Kownacki E, Eder M, Degiacomi P, Zoller N, Devay J, Lange J, Schnider T: Factor XIII substitution in surgical cancer patients at high risk for intraoperative bleeding. Anesthesiology 2009;110:239-245.

16 Godje O, Haushofer M, Lamm P, Reichart B: The effect of factor XIII on bleeding in coronary surgery. Thorac Cardiovasc Surg 1998;46:263-267.

17 Godje O, Gallmeier U, Schelian M, Grunewald M, Mair H: Coagulation factor XIII reduces postoperative bleeding after coronary surgery with extracorporeal circulation. Thorac Cardiovasc Surg 2006;54:26-33.

18 Grothaus-Pinke B, Gunzelmann S, Fauser AA, Kiehl MG: Factor XIII replacement in stem cell transplant (SCT) recipients with severe graft-versus-host disease of the bowel: report of an initial experience. Transplantation 2001;72:1456-1458.

19 Demesmay K, Tissot E, Bulabois CE, Bertrand MA, Racadot E, Woronoff-Lemsi MC, Cahn JY, Deconinck E: Factor XIII replacement in stem-cell transplant recipients with severe hemorrhagic cystitis: a report of four cases. Transplantation 2002;74:1190-1192.

20 Reid PC, Coker A, Coltart R: Assessment of menstrual blood loss using a pictorial chart: a validation study. BJOG 2000;107:320-322.

21 Szanto T, Salmela B, Makipernaa A, Armstrong E, Lassila R: Management of acute myocardial infarction in a patient with factor XIII deficiency using prophylactic factor replacement therapy. Haemophilia 2008; 14:163-165.

22 Boehlen F, Casini A, Chizzolini C, Mansouri B, Kohler HP, Schroeder V, Reber G, de Moerloose P: Acquired factor XIII deficiency: a therapeutic challenge. Thromb Haemost 2013;109:479-487.

23 Jantsch-Plunger V, Beck G, Maurer W: PCR detection of a low viral load in a prothrombin complex concentrate that transmitted hepatitis B virus. Vox Sang 1995; 69:352-354.

24 Matayoshi T, Omi T, Sakai N, Kawana S: Clinical significance of blood coagulation factor XIII activity in adult Henoch-Schonlein purpura. J Nippon Med Sch 2013;80:268-278.
Brackmann HH, Egbring R, Ferster A, Fondu P, Girardel JM, Kreuz W, Masure R, Miloszewski K, Stibbe J, Zimmermann R, et al.: Pharmacokinetics and tolerability of factor XIII concentrates prepared from human placenta or plasma: a crossover randomised study. Thromb Haemost 1995;74:622-625.

26 Bregenzer N, Caesar I, Andus T, Hamling J, Malchow $\mathrm{H}$, Schreiber S, Scholmerich J: Lack of clinical efficacy of additional factor XIII treatment in patients with steroid refractory colitis. The Factor XIII Study Group. Z Gastroenterol 1999;37:999-1004.

27 Nugent DJ: Prophylaxis in rare coagulation disorders - factor XIII deficiency. Thromb Res 2006;118(suppl 1):S23-28.

28 Ashley C, Chang E, Davis J, Mangione A, Frame V, Nugent DJ: Efficacy and safety of prophylactic treatment with plasma-derived factor XIII concentrate (human) in patients with congenital factor XIII deficiency. Haemophilia 2015;21:102-108.

29 Anwar R, Miloszewski KJ: Factor XIII deficiency. Br J Haematol 1999;107:468-484.

30 Muszbek L, Bagoly Z, Cairo A, Peyvandi F: Novel aspects of factor XIII deficiency. Curr Opin Hematol 2011;18:366-372.

31 Acharya SS, Coughlin A, Dimichele DM, North American Rare Bleeding Disorder Study Group: Rare bleeding disorder registry: deficiencies of factors II, V, VII, $\mathrm{X}, \mathrm{XIII}$, fibrinogen and dysfibrinogenemias. J Thromb Haemost 2004;2:248-256.

32 Groener A, Nowak T, Popp B, Schaefer W: A plasmaderived FXIII concentrate with a very high margin of virus safety for the treatment of congenital FXIII deficiency. XXIII Congress of the International Society on Thrombosis and Haemostasis 57th Annual SSC Meeting, Kyoto, Japan. J Thromb Haemost 2011;9(suppl 2): 222.

33 Velthove KJ, Over J, Abbink K, Janssen MP: Viral safety of human plasma-derived medicinal products: impact of regulation requirements. Transfus Med Rev 2013;27:179-183.

34 Narick C, Triulzi DJ, Yazer MH: Transfusion-associated circulatory overload after plasma transfusion. Transfusion 2012;52:160-165.

35 Yang L, Stanworth S, Baglin T: Cryoprecipitate: an outmoded treatment? Transfus Med 2012;22:315-320.

36 Jacob D, Marron B, Ehrlich J, Rutherford PA: Pharmacovigilance as a tool for safety and monitoring: a review of general issues and the specific challenges with end-stage renal failure patients. Drug Healthc Patient Saf 2013;5:105-112. 